\title{
Stellenwert der endoskopischen Axilladissektion beim invasiven Mammakarzinom
}

\author{
M. Zuber, T. Kocher, I. Langer und F. Harder
}

Schlïsselwörter: Mammakarzinom - Axilla - weniger invasive
Chirurgie - endoskopische Axilladissektion.

Kevwords: Breast cancer-axilla-less invasive surgery-endoscopic axillary dissection.

Zusammenfassung: Grundlagen: Die Axilladissektion liefert zuverlässige prognostische Information. bestimmt mehrheitlich die adjuvante Therapie und reduziert die axillären Tumorrezidive. Die Morbidität nach Axilladissektion kann jedoch nicht vernachlässigt werden (sensible Störungen, Schmerzen, motorische Schwäche, Lymphödem). Patientinnen mit kleinen Tumoren (pTla, b, c) könnten von neueren weniger invasiven Verfahren (endoskopische Axilladissektion) oder von selektiveren Methoden (Sentinel-lymph-node-Procedere) profitieren. In dieser prospektiven Studie wurde die axilloskopische Lymphadenektomie evaluiert.

Methodik: 55 klinisch nodal negative Patientinnen (Durchschnittsalter: 60 Jahre [ 30 bis 86 Jahre]) wurden von einem Chirurgen endoskopisch operiert (Januar 1996 bis Juni 1998). Nach Liposuktion des axillären Fettkörpers wurden die Lymphknoten von Level I + II identifiziert und unter direkter endoskopischer Sicht reseziert (erfolgreiches Verfahren in $95 \%: n=52$ ). Die Patientinnen wurden entsprechend dem Nachsorgeschema alle 4 Monate kontrolliert. Nach einer mittleren Beobachtungszeit von 22 Monaten ( 7 bis 37 Monate; $n=51$ [eine Patientin verweigerte die Nachsorge]) wurden die Patientinnnen mit einem Evaluationsfragebogen, einem Interview und einer klinischen Untersuchung (inklusiv Schultergelenksfunktion, Zirkumferenzmessungen der oberen Extremität) nachkontrolliert.

Ergebnisse: Durchschnittlich wurden 13,3 (5 bis 25) Lymphknoten endoskopisch entfernt. Nodal positive Lymphknoten wurden in $31 \%(n=16)$ beobachtet. Der Durchschnittswert der positiven Lymphknoten/Patientin betrug 3,1 (1 bis 10). Acht Serome $(15 \%)$ mußten in der frühpostoperativen Phase punktiert werden. Es traten keine Hämatome, jedoch ein Infekt in der Axilla nach Chemotherapie auf $(2 \%)$. Nach einer mittleren Beobachtungszeit von 22 Monaten ( 7 bis 37 Monate) konnten keine axillären Rezidive nachgewiesen werden. Eine subkutane Implantationsmetastase in der Narbe eines Trokarkanals (1/55, $2 \%$ ) wurde diagnostiziert und reseziert. Klinisch traten keine Lymphödeme auf. Die frühe Mobilisation des Schultergelenkes war subjektiv und objektiv gut.

Schlußfolgerungen: Die durchschnittlich 13 axilloskopisch entfernten Lymphknoten entsprechen der Anzahl resezierter Lymphknoten bei offener Axilladissektion. Die Morbidität dieses in Evaluation begriffenen Verfahrens ist niedrig. Nach einer mittleren Beobachtungszeit von 22 Monaten wurde ein Implantationsrezidiv im subkutanen Kanal des anterioren Arbeitstrokar nachgewiesen. Eine längere Beobachtungszeit ist nötig, um die Technik empfehlen zu können. Es zeichnet sich ab, daß die endoskopische, axilläre Lymphknotendissektion durch das selektivere Sentinel-lymph-node-Verfahren abgelöst wird.

(Acta Chir. Austriaca 2000; 32: 110-114)

Korrespondenzanschrift: PD Dr. M. Zuber, Departement Chirurgie der Universität Basel, Allgemeinchirurgische Klinik, Spitalstraße 21, CH-4031 Basel, Schweiz.

Fax: ++41/61/265-7796

E-mail: mzuber@uhbs.ch

\section{Endoscopic Axillary Dissection and its Perspective in Breast Cancer}

Summary: Background: Axillary clearance provides prognostic information, determines adjuvant therapy and reduces axillary recurrences. However, significant morbidity may follow axillary dissection. Patients with small tumors (pTla, b, c) may benefit from newer less invasive (endoscopic lymph node dissection) or more selective (sentinel lymph node biopsy) axillary procedures in evaluation since the early nineties. In this prospective study the axilloscopic approach was evaluated.

Methods: 55 clinically node negative patients (mean age: 60 years [30 to 86 years]) had surgery by one single surgeon (January 1996 to June 1998). After axillary liposuction individual lymph nodes (level I + II) were identified and removed under direct vision by means of a laparoscope (successful procedure in $95 \%: \mathrm{n}$ $=52$ ). Patients were followed every four months according to a institution-based follow-up protocol. Results of 51 patients (1 refused) were analyzed after a median follow-up of 22 (7 to 37) months by self-evaluation questionnaire, interview and clinical examination (including: range of motion of the shoulder joint, circumferences of the upper extremities).

Results: An average of 13.3 (5 to 25 ) lymph nodes was endoscopically removed. Patients $(n=16 ; 31 \%)$ had involved nodes with a mean of 3.1 nodes positive per individuum. Seromas necessitated needle aspiration in 8 patients $(15 \%)$. There were no hematomas and one low-grade infection $(2 \%)$. After a median follow up period of 22 ( 7 to 37 ) months no axillary relapse but one trocar site implantation metastasis $(1 / 55,2 \%)$ was detected and resected. No lymph edema was observed. Early range of motion of the shoulder joint was excellent.

Conclusions: 13 endoscopically removed lymph nodes on an average comes close to numbers reported in open axillary surgery. Morbidity is low. After a median follow-up of 22 months 1 trocar site metastasis was observed and excised. A longer follow-up time and randomized protocols are mandatory until this endoscopic method can be recommended. More selective procedures in evaluation such as the sentinel lymph node biopsy may have an impact on the value of the axilloscopic surgery in the treatment of breast cancer patients.

\section{Einleitung}

Die axilläre Lymphadenektomie ist integraler Bestandteil der Chirurgie des invasiven Mammakarzinoms. Die En-bloc-Resektion der tumordrainierenden axillären Lymphknoten bei maligne erkrankter Brust ist beispielhaft für die onkologische Chirurgie.

Die Axilladissektion liefert zuverlässige prognostische Information, bestimmt mehrheitlich die adjuvante Therapie für kleine Karzinome ( $\leqq 1 \mathrm{~cm})$ und kann zu einer regionären Tumorkontrolle im Sinne einer axillären Rezidivprophylaxe führen (2). Wird eine Axilladissektion durchgeführt, so ist die axilläre Rezidivrate bei 0 bis $2,1 \%(17,20,28)$. Erfolgt keine axilläre Lymphadenektomie, so beträgt die Rezidivhäufigkeit in der Achselhöhle zwischen 3,5 und $28 \%$ je nach Tumorgröße und Nachsorgezeit (1, $14,20,38$ ). Eine auswertbare, randomisierte Studie zur Überprïfung der Wertigkeit der Axilladissektion versus Beobachtung existiert nicht (5).

Die Morbidität nach offener, axillärer Lymphadenektomie von Level I + II nach Berg kann nicht außer Acht gelassen werden $(18,23)$. Neben sensiblen Störungen und Schmerzempfindungen am Oberarm ist es die eingeschränkte Funktion im Schultergelenk und vor allem das handikapierende Lymphödem unterschiedlichen Schweregrades der oberen Exrtremität, wel- 
che heute im Rahmen des Therapiekonzeptes der kleineren Mammakarzinome neu bewertet werden $(16,23,24)$. In der $\mathrm{Li}$ teratur wird über das Armlymphödem nach chirurgischer Dissektion (ohne Strahlentherapie für die Axilla) in 2 bis $24 \%$ (29. 30,37 ) berichtet.

Auf Grund der Intensivierung der sekundären Präventionsmaßnahmen (Sensibilisierung der Frauen, Selbstuntersuchung, Mammographie, mammographisches Screening) werden häufiger Primärtumoren mit kleineren Durchmesser entdeckt $(6,7$, 22). Das Risiko axillärer Lymphknotenmetastasen ist direkt abhängig von der Tumorgröße. Mammographisch entđeckte pTla-Tumoren (bis $5 \mathrm{~mm}$ ) weisen in 0 bis $7 \%$ und pTlb ( $>5$ bis $10 \mathrm{~mm}$ ) in 7 bis $17 \%$ histopathologisch positive Lymphknoten auf $(29,40)$. Tumoren mit Durchmesser $z$ wischen 10 und $20 \mathrm{~mm}$ (pTlc) sind häufiger nodal positiv (bis $33 \%)(9,11,32,40)$.

Multivariate Analysen von Patientinnen- und Primärtumorcharakteristika ergeben eine zu ungenaue Prädiktion des axillären Nodalstatus (27). Die Korrelation von axillärem Ultraschall und Lymphknoten-Feinnadelzytologien beträgt $63 \%$ (3). Die Korrelation zwischen PET und axillärem Lymphknotenbefall betrug in einer hervorragenden Arbeit $95 \%$ (33). In anderen Arbeiten ist dieser Wert sehr viel tiefer angesetzt. Auch wenn die moderne Bildgebung Entwicklungspotential in sich birgt, so sind heute praktikable neue Standardverfahren zur Identifikation des axillären Nodalstatus gefragt. Nachdem Ende der achtziger Jahre die Indikation zur systemischen Chemotherapie auf Grund von Primärtumorfaktoren unabhängig vom Befund der axillären Lymphknoten erweitert worden ist (13), wurde die Morbidität der Axilladissektion neu bewertet. Dies hatte zur Folge, daß zu Beginn der neunziger Jahre innovative, weniger invasive Verfahren für die Axilla evaluiert wurden (21). Eine der neuen Methoden ist das beim Melanom (25) entwickelte und fur das Mammakarzinom (11) übernommene Sentinel-lymph-node (SLN)Verfahren. Die endoskopische Axilladissektion ist die zweite Methode, welche neu entwickelt wurde $(31,34)$

\section{Patientinnen und Methoden}

\section{Patientinnen und Operationen}

Das Durchschnittsalter der 55 Patientinnen mit klinisch unauffälliger Axilla betrug 60 Jahre ( 30 bis 86 Jahre). Das Verhältnis zwischen prä- und postmenopausalem Status war 10:40. Die histopathologische Messung des Primärtumor-Durchmessers ergab im Durchschnitt $22 \mathrm{~mm}$ ( 3 bis $50 \mathrm{~mm}$; $\mathrm{n}=52$ ). Mehrheitlich handelte es sich um invasive duktale (39) und lobuläre (8) Karzinome. Die endoskopischen, axillären Lymphadenektomien wurden von einem Operateur zwischen Januar 1996 bis Juni 1998 durchgeführt. Erfolgreich gelang das axilloskopische Verfahren in 52 Fällen $(95 \%)$. Bei 3 Patientinnen $(5 \%)$ mußte zu einem offenen Verfahren konvertiert werden: Orientierungsprobleme in einer adipösen Axilla ( $1 \mathrm{x})$ und bei einer Blutung aus der $\mathrm{V}$. thoracodorsalis $(1 \mathrm{x})$, sowie wegen eines derb veränderten axillären Fettkörpers nach neoadjuvanter Chemotherapie (1x).

Technik der endoskopischen axillären Lymphknotendissektion

Eine Gruppe von Gynäkologen aus Clermont-Ferrand/Frankreich haben 1994 über Erfahrungen mit der axillären Liposuktion berichtet (34). Dabei wird der axilläre Fettkörper mit Liposuktionslösung infiltriert und anschließend abgesaugt. Über eine ca. $3 \mathrm{~cm}$ lange Inzision kann ein ,Lymphknoten-Picking“ angeschlossen werden. Die Methode der axillären Liposuktion wurde im folgenden durch die axilloskopische Dissektion erweitert. Das Verfahren ist detailliert beschrieben worden $(21,34,39)$.

\section{Instrumentarium}

Für den Mammaeingriff:

- Instrumente für die Tumorektomie

Für die axilläre Liposuktion:

- $1 \times 20$-ml-Injektionsspritzen mit langen und kurzen Kanülen

- Liposuktionslösung (500 ml 0,45\% NaCl, $10 \mathrm{ml} 4 \%$ iges Lidocain, $5 \mathrm{mg}$ Adrenalin)
- Abortkanüle (No. 7) mit abgerundetem Ende und seitlicher Öffnung

- Handgriff mit Schiebeventil zur Unterdruckkontrolle

- Liposuktionspumpe (Unterdruck 800 - maximal 1000 mbar)

Für die endoskopische Axilladissektion:

- Videoturm mit Monitor, Lichtquelle. Kamerasteuerung und $\mathrm{CO}_{2}$-Insufflator

- 10-mm-Winkeloptik (25) mit Kamera und Lichtkabel

- 5-mm-Arthroskopieoptik

- $1 \times 10$-mm-, $2 \times 5$-mm-Trokare

- $1 \times$ ca. $20 \mathrm{~cm}$ lange $5-\mathrm{mm}-S c h e r e$ mit Diathermieanschluß

- $1 \times$ ca. $20 \mathrm{~cm}$ lange $5-\mathrm{mm}$-Faßzange

- Diathermiegerät

Operationstechnik

Die Operation wird in Rückenlage und Intubationsnarkose durchgeführt (Antibiotikumprophylaxe: $1.5 \mathrm{~g}$ Cefuroxime i.v. [Zinacef ${ }^{\circledR}$ ], Einmaldosis). Der Arm wird in Abduktionsstellung frei beweglich ausgelagert.

1. Instillation der Liposuktionsflïssigkeit: Der axilläre Fettkörper wird erst oberflächlich, dann tief innerhalb der Grenzen der Axilla mit Liposuktionsfliissigkeit instilliert. Je nach Fettgehalt werden mit mehreren Punktionen 250 bis 450 ml Liposuktionslösung injiziert. Eine Gefäßpunktion muß vermieden werden. Durch zusätzliches Einmassieren wird für eine optimale Verteilung der Flüssigkeit gesorgt.

2. Tumorektomie: Während die Liposuktionslösung einwirkt, wird die Tumorektomie durchgeführt. Eine axillanahe Tumorlage ist für die axilloskopische Dissektion ungeeignet wegen der Interferenz von Tumor und ventralem Arbeitstrokar.

3. Liposuktion: Etwa 20 Minuten nach Instillation wird die Haut ca. $12 \mathrm{~cm}$ kaudal der Axillarfalte in der mittleren Axillarlinie 10 bis $15 \mathrm{~mm}$ lang inzidiert. Mit einer Klemme wird in den axillären Fettkörper eingegangen und gespreizt. Durch diese Öffnung wird der Liposuktionssauger eingeführt. Das axilläre Fett wird durch gleichmäßiges Hin- und Herbewegen des Saugrohres bei 800 mbar Unterdruck aspiriert. Fächerförmig wird das aufgelockerte Fett von der Obertläche kommend bis in die tieferen Schichten abgesaugt. Die Liposuktion wird so lange fortgeführt, bis das Aspirat lachsfarben und schaumig wird. Dies dauert in der Regel 10 bis 15 Minuten.

4. Endoskopische Axilladissektion: Durch die bereits bestehende Öffnung wird ein 10-mm-Trokar gasdicht eingeführt und mit einem Faden an der Haut fixiert. Die Axilla wird dann bis auf $8 \mathrm{~mm} \mathrm{Hg}$ mit $\mathrm{CO}_{2}$ insuffliert. Zwei weitere Zugänge $(5 \mathrm{~mm})$ werden unter videoendoskopischer Kontrolle je $10 \mathrm{~cm}$ kaudal der Axillarfalte in der vorderen und hinteren Axillarlinie plaziert. Unter Schonung des thorakodorsalen Gefäßnervenbündels, des $\mathrm{N}$. thoracicus longus und des $\mathrm{N}$. pectoralis lateralis werden nun mit Faßzange und Schere die Lymphknoten von Level I + Il disseziert. Einzelne kleine Lymphknoten werden durch die 5-mm-Trokare entfernt. Die meist größeren Lymphknotenpakete werden vor dem Kameratrokar zur späteren Extraktion deponiert. Ist die Dissektion beendet, wird auf die 5-mm-Optik gewechselt und die Lymphknoten werden durch den 10-mmTrokar extrahiert. Das Operationsfeld wird gespült. Eine Drainage wird nicht eingelegt. Die Wunden werden abgepolstert und mit einer elastischen Brustbinde leicht komprimiert.

5. Filtrierung des Liposuktionsaspirates: Zum Schluß wird die Liposuktionsflüssigkeit durch eine Gazekompresse filtriert. Aspirierte Lymphknoten werden aufgesucht, asserviert und separat von den endoskopisch resezierten Lymphknoten histopathologisch aufgearbeitet.

\section{Nachsorge}

Die Patientinnen wurden entsprechend einem Nachsorgeschema alle 4 Monate kontrolliert. Nach einer mittleren Beobachtungszeit von 22 Monaten ( 7 bis 37 Monate) wurden 51 Patientinnen mit einem Evaluationsfragebogen, einem Interview und einer klinischen Untersuchung nachkontrolliert. Die Untersuchung umfaßte auch eine Schultergelenksfuntionsüberprüfung und eine Zirkumferenzmessung an Unter- und Oberarm (maximale 


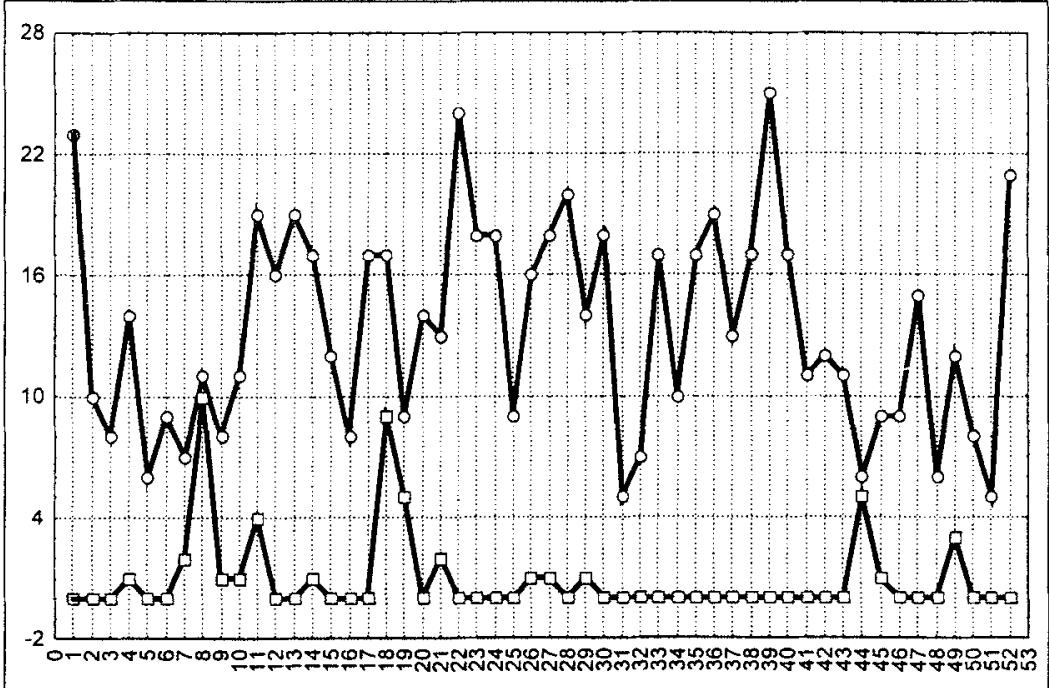

Abb. 1. Total resezierte Lymphknoten und Anzahl positiver Lymphknoten ( $n=52$ ). Obere Kurve: Total der resezierten Lymphknoten in chronologischer Ordnung der 52 Patientinnen mit endoskopischer axillärer Lymphadenektomie (Durchschnitt 13,3 15 bis 25] Lymphknoten). Untere Kurve: Anzahl positiver Lymphknoten für jeden individuellen Fall (pN+: 16/52 [31\%], durchschnittlich 3,1 positive Lvmphknoten/Individuum).

Umfänge). Eine Patientin, die sich selbst einen unauffälligen Verlauf attestierte, verweigerte eine detaillierte Nachsorge. Die 3 Patientinnen, bei welchen intraoperativ auf ein offenes Verfahren umgestiegen werden mußte, sind ebenfalis nachgesorgt worden.

\section{Ergebnisse}

Im Durchschnitt wurden bei 52 Patientinnen 13,3 (5 bis 25) Lymphknoten endoskopisch entfernt (Tab. 1, Abb. 1). In den ersten 10 Fällen waren es durchschnittlich 11 Lymphknoten. Dieser Durchschnitt erhöhte sich nur leicht in den Fällen 11 bis 52. Die Liposuktion förderte durchschnittlich 2 Lymphknoten (I bis 9). 16 Patientinnen ( $31 \%$ ) waren nodal positiv; durchschnittlich ergaben sich 3,1 positive Lymphknoten/Patientin ( 1 bis 10). Eine Verletzung des $\mathrm{N}$. thoracodorsalis führte zu einer persitierenden Scapula alata. 8 Serome $(15 \%)$ mußten früh postopertiv punktiert werden. Es kamen keine Hämatome vor. Nach einem ersten Zyklus Chemotherapie wurde 1 Infekt in der Axilla manifest und mußte chirurgisch revidiert werden. Es dürfte sich um einen Low-grade-Infekt gehandelt haben, der sich weder subjektiv noch klinisch manifestierte und erst unter Chemotherapie evident wurde. Nach Ausheilung der Infektion konnte die Systemtherapie weitergeführt werden. Residuen im Sinne von Schmerzen, Bewegungseinschränkungen im Schultergelenk oder eine Schwellung des Armes sind bei dieser Patientin nicht zu verzeichnen.

Die Patientinnen wurden im Durchschnitt 5,6 (1 bis 16) Tage postoperativ entlassen (Tab. 1). Jüngere Patientinnen unter 50 Jahre waren postoperativ im Mittel nach 4,6 (1 bis 8 ) Tagen wieder zu Hause.

Nach einer mittleren Beobachtungszeit von 22 (7 bis 37) Monaten war kein axilläres Rezidiv nachzuweisen, jedoch fiel eine Implantationsmetastase im subkutanen Kanal eines anterioren Arbeitstrokars auf. Die Metastase wurde feinnadelbioptisch verifiziert und im Gesunden reseziert. Weitere regionäre Tumorrezidive sind nicht aufgetreten. 3 Patientinnen sind an ihrem metastasierenden Leiden gestorben $(1 \times$ Lebermetastasen 11 Monate postopertiv, primär pT2 pN0 cM0; $1 \times$ Lungen-, Leber-, Hirnmetastasen 14 Monate postoperativ, primär pT2 $\mathrm{pN} 1 \mathrm{cMo} ; 1 \times$ Knochen-, Lungen- Hirnmetastasen 20 Monate postoperativ, primär pT2 pN0 cM0). Bei 2 weiteren Patientinnen sind Fernmetastasen bekannt $(1 \times$ Knochen, $1 \times$ Lunge $)$.
Alle 51 Patientinnen haben den Selbstevaluations-Fragebogen ausgefüllt. Die Ergebnisse sind in Tab. 2 wiedergegeben.

Die Funktionsmessung im Schultergelenk nach der Null-Durchgangsmethode ergab in 49 Fällen eine seitengleiche, normale Funktion. In 2 Fällen resultierte eine eingeschränkte Schulterbeweglichkeit: $1 \times-20^{\circ}$ Anteversion, $1 \times-30^{\circ}$ Abduktion). Diese beiden Patientinnen waren durch diesen Befund im täglichen Leben gestört. Nacken- und Schürzengriff waren in beiden Fällen möglich. Die Umfangdifferenz am Oberarm betrug im Durchschnitt $0,17 \mathrm{~cm}(-2$ bis $+3 \mathrm{~cm})$ am Unterarm $-0,1 \mathrm{~cm}(-2$ bis +2$)$. Die klinische Untersuchung ergab unter Einbezug der physiologischen Seitendifferenzen (dominante Seite) keine Anhaltspunkte für Lymphödeme.

Das Patientinnenkollektiv wird entsprechend dem Nachsorgeschema weiter kontrolliert.

\section{Diskussion}

Während für größere Primärkarzinome und klinisch verdächtige Axillae die offene axilläre Lymphadenektomie für alle Risiko- und Altersgruppen allgemein akzepiert ist, besteht ein $\mathrm{Di}$ lemma über die Notwendigkeit der Axilladissektion für kleine oder nicht palpable Tumoren bei $\mathrm{klinisch}$ unauffälliger Achselhöhle. Es sind die Patientinnen mit den kleinen Läsionen, für welche das axilläre Lymphknoten-Staging wegweisend ist für die Indikation einer systemischen Chemotherapie. Ist in dieser Situation eine formale Dissektion von Level I + II gerechtfertigt, wenn in der Mehrheit dieser Fälle ein histopathologisch negatives Nodalstadium resultiert? Für die Patientinnen stellt dieses Vorgehen keinen Vorteil dar, da insbesondere dieses Procedere mit einem erhöhten Risiko der Langzeitmorbidität vergesellschaftet ist.

Diese Situation zu Beginn der neunziger Jahre ließ die Entwicklung weniger invasiver (endoskopische Dissektion) und selektiverer (SLN-Biopsie) axillärer Verfahren zu (18). Solange

\section{Tab. 1. Charakteristika der Patientinnen $(n=52)$.}

\begin{tabular}{|l|c|c|c|}
\hline Durchschnittsalter & 60 & $30-86$ & Jahre \\
Prä- : postmenopausal Status & $10: 40$ & & \\
Mittlere postopertive Hospitalisationszeit & 5,6 & $1-16$ & Tage \\
Patientinnen < 50 Jahre & 4,6 & $1-8$ & Tage \\
Durchschnittlich entfernte Lymphknoten & 13,3 & $5-25$ & \\
Liposuktion (Lymphknoten) & 2 & $0-9$ & \\
Anzahl Patientinnen mit pN+ Status & $16 / 52$ & $31 \%$ & \\
Anzahl positive Lymphknoten/Fall & 3,1 & $1-10$ & \\
\hline
\end{tabular}

Tab. 2. Selbstevaluation der Patientinnen $(n=51$; Nachsorge verweigert $n=1$ ).

\begin{tabular}{|l|c|c|}
\hline \multirow{2}{*}{} & \multicolumn{2}{|c|}{ Subjektiver Wert* } \\
\cline { 2 - 3 } & Durchschnitt & Umfang \\
\hline Schmerzen Oberarm & 9,4 & $4-10$ \\
Schmerzen Unterarm & 9,9 & $6-10$ \\
Schmerzen Brust/Brustwand & 9,2 & $6-10$ \\
Bewegungsausmass Schultergelenk & 9,4 & $4-10$ \\
Gebrauchsfähigkeit im Alltag & 9,5 & $5-10$ \\
\hline
\end{tabular}

* Skala 1-10: 1 = sehr schlecht; $10=$ exzellent 
Tab. 3. Ausschilusskriterien fïr die endoskopische Axilladisvektion.

- Verdächtige axilläre Lymphknoten (Tumorzelldissemination)

- Adipöse Axilla (Orientierung)

- St. n. neoadjuvanter Chemotherapie (Fettgewebeveränderung)

- Tumor lateral im Axillarfortsatz der Brustdrüse (Interferenz mit Arbeitstrokar)

keine molekularbiologischen Faktoren des Primärtumors entdeckt oder sensitive und spezifische bildgebende Verfahren definiert sind, die den Nodalstatus respektiv die biologische Aggressivität zuverlässig voraussagen können, müssen innovative Methoden mit niedriger Morbidität und ausgezeichneter Kosmetik evaluiert werden. Diese Voraussetzungen treffen auf die endoskopische axilläre Lymphadenektomie zu.

Die axilloskopische Dissektion bricht mit einem allgemein akzeptierten onkochirurgischen Prinzip: der En-bloc-Resektion. Welchen Einfluß hat die Mißachtung dieser Regel durch potentiell provokative Manipulationen (Instillation, Liposuktion, endoskopisches Operieren) für eine Tumorzelldissemination auf die axilläre Rezidivhäufigkeit und Frequenz von Implantationsmetastasen in Trokarkanälen?

Axilläre Rezidive nach endoskopischer Lymphadenektomie sind in der Literatur nur in einer Arbeit beschrieben (31). In dieser Studie wurde die Axilloskopie (20 Fälle) versus die offene Technik (20 Fälle) randomisiert. Nach einer kurzen Beobachtungszeit sind in der endoskopischen Gruppe $2(10 \%)$ axilläre Rezidive beobachtet und behandelt worden. Nach einer mittleren Nachsorgezeit von 22 ( 7 bis 37) Monate wurde in der vorliegenden Serie von 52 Patientinnen eine Trokar-Implantationsmetastase $(2 \%)$ festgestellt und therapiert (Tab. 5). Es handelte sich in diesen 3 Rezidivfällen $(2+1)$ um pN 1 -Stadien. In allen publizierten Axilloskopie-Protokollen ist die Beobachtungszeit zu kurz, um die Wertigkeit des endoskopischen Verfahrens in Bezug auf die Tumorprogression abschließend zu beurteilen.

Im Gegensatz zu Suzanne (Suzanne 1998, persönliche Mitteilung), der keine Unterschiede im Schweregrad der morphologischen Läsionen (Hämorrhagie, Kapseleinrisse, Zerreißungen und Fragmentierung) von Lymphknoten nach Entfernung mit- tels offener oder axilloskopischer Technik feststellen konnte, beobachtete Salvat zweimal häufiger morphologische Defekte an den Lymphknoten. jedoch war die Differenz statistisch nicht signifikant (31). Bei Brun wiesen 21 der 50 positiven Lymphknoten Kapseleinrisse auf (4). Es bedarf weiterer Untersuchungen (2. B. Spülzytologie, rt-PCR [26]), um residuelle Karzinomzellen nach Abschluß der endoskopischen Axilladissektion nachzuweisen. Denn diese epithelialen Elemente könnten die Grundlage für axilläre Rezidive sein.

Die Vorteile der Liposuktion und endoskopischen Axilladissektion sind die geringere Invasivität, die verminderte Morbidität, das gute kosmetische Resultat und die hohe Akzeptanz durch die Patientinnen (Tab. 4). Zusätzlich hat diese Methode gezeigt, daß mit der Anzahl der resezierten Lymphknoten dieselbe prognostische Information (Tab. 5) wie beim offenen Staging erreicht wird. Die Ausschlußkriterien müssen beachtet werden (Tab. 3). Langzeitresultate dieses Verfahrens stehen noch aus.

Es ist nicht der Mangel an langjähriger Erfahrung mit dieser endoskopischen Methode, sondern vielmehr die überwiegenden Vorteile des SLN-Verfahrens, welches das letztere attraktiver erscheinen läßt. Die SLN-Methode $(11,40)$ ist eindeutig noch weniger invasiv, praktikabler für eine breite Anwendung und schneller durchfuhrbar. Das Verfahren hat die Potenz, das zukünftige Selektionskriterium für die Indikation zur Axilladissektion zu werden.

Ob die Axilloskopie komplementär bei histologisch positivem SLN eingesetzt werden soll, scheint uns heute fragwürdig. Denn erstens, ist es onkochirurgisch bei bewiesenem positivem (SLN-) Nodalstatus zu rechtfertigen, eine endoskopische Methode in Evaluation anzuwenden? Zweitens, ist die Axilloskopie nach Voroperation (SLN-Procedere) komplexer. Eine Indikation für das endoskopische Verfahren könnte bei histologisch nachgewiesenen Mikrometastasen im SLN überprüft werden, weil bei pT1-Tumoren nur in $6 \%$ weitere Non-SLN tumorbefallen sind (8). In diesen Fällen kann die Gefahr einer möglichen Tumorzelldissemination als gering eingestuft werden.

Machbar ist auch die axilloskopische Detektion und Biopsie des SLN (36). Endoskopische Gammadetektoren sind auf dem Markt erhältlich. Der Material- und Zeitaufwand ist aus eigener Erfahrung enorm und der Vorteil für die Patientinnen im Vergleich zur offenen SLN-Technik kaum erheblich.
Tab. 4. Vor- und Nachteile der endoskopischen Axilladissektion.

\begin{tabular}{|l|l|}
\hline Vorteile & Nachteile \\
\hline - Gute Sicht & - Nicht konform mit onkochirur- \\
gischem Prinzip (en bloc Resektion)
\end{tabular}

Tab. 5. Endoskopische axilläre Lymphadenektomie: Laufende publizierte Studien.

\begin{tabular}{|lcc|c|c|l|c|}
\hline Studie* & $\begin{array}{c}\text { Axilloskopien } \\
\mathbf{n}\end{array}$ & $\begin{array}{c}\varnothing \mathbf{L K} \\
\mathbf{n}\end{array}$ & $\begin{array}{c}\varnothing \mathbf{L K} \\
\mathbf{n}\end{array}$ & $\begin{array}{l}\text { Zeit seit } \\
\text { Studienbeginn }\end{array}$ & $\begin{array}{l}\text { Axilläre } \\
\text { Residive }\end{array}$ \\
\hline Suzanne & $\mathbf{1 9 9 6}$ & 159 & 14 & 7 & $\cong 5$ Jahre & $?$ \\
Salvat & $\mathbf{1 9 9 6}$ & 20 & 12,9 & $?$ & $\cong 3,2$ Jahre & $210 \%$ \\
Brun & $\mathbf{1 9 9 8}$ & 43 & 13,6 & 2,5 & $\cong 3,4$ Jahre & $?$ \\
Basel & $\mathbf{1 9 9 9}$ & 52 & 13,3 & 2,2 & $\equiv 3$ Jahre & $12 \%$ \\
\hline
\end{tabular}

* vgl. Literaturangaben

\section{Literatur}

(1) Baxter N. McCready D. Chapman JA. Fish E. Kahn H. Hanna W, Trudeau M, Lickley HL: Clinical behavior of untreated axillary nodes after local treatment for primary breast cancer. Ann Surg Oncol 1996; 3:235. (2) Bonnema J. van Geel AN, van Ooijen B, Mali SPM, Tjiam SL. Henzen-Logmans SC. Schmitz PIM, Wiggers T: Ultrasound-guided aspiration biopsy for detection of nonpalpable axillary node metastases in breast cancer patients: New diagnostic method. World J Surg 1997; 21 : 270 .

(3) Bland KI. Scott-Conner CEH, Menck H, Winchester DP: Axillary dissection in breast-conserving surgery for stage I and II breast cancer: $\mathrm{A}$ national cancer data base study of patterns of omission and implication for survival. J Am Coll Surg 1999; 188: 586.

(4) Brun JL. Rousseau E. Bełleannée, de Mascarel A, Brun G: Axillary lymphadenectomy prepared by fat and lymph node suction in breast cancer. Eur J Surg Oncol 1998; 24: 17.

(5) Cady B. Stone MD, Schuler JG, Thakur R, Wanner MA, Lavin PT: The new era in breast cancer: Invasion, size, and nodal involvement dramatically decreasing as a result of mammographic screening. Arch Surg 1996; 131: 307.

(6) Cady B: Basic principles in surgical oncology. Arch Surg 1997; 132: 338.

(7) Cabanes PA, Salmon RJ, Vilcoq JR, Durand JC, Fourquet A, Gautier $C$, Aselain $B$ : Value of axillary dissection in addition to lumpectomy and radiotherapy in early breast cancer. Lancet 1992; 339: 1245 .

(8) Chu KU, Turner RR, Hansen NM, Brennan MB, Bilchik A, Giuliano $\mathrm{AE}$ : Do all patients with sentinel lymph node metastasis from breast cancer need complete axillary dissection? Ann Surg 1999; 229: 536.

(9) Dowlatshahi K, Snider HC, Kim R: Axillary node status in nonpalpable breast cancer. Ann Surg Oncol 1995; 2: 424.

(10) Giard S. Laurent JC, Dron AM, Lefebre D: Lymphadénectomie axillaire préparée par lipoaspiration axillaire contre curage axillaire functionnel: Résulıts préliminaires d'un essai prospectif randomisé. Bull Cancer 1997: 8+: 254

(11) Giuliano AE, Kirgan DM, Guenther JM, Morton DL: Lymphatic mapping and sentinel lymphadenectomy for breast cancer. Ann Suro 1994; $220: 391$. 
(12) Giuliano AE. Barth AM. Spivack B, Beitsch PD. Evans SW: Incidence and predictors of axillary metastasis in T1 carcinoma of the breast. J Am Coll Surg 1996: 183: 185 .

(13) Greco M. Agresti R. Raselli R. Giovanazzi R. Veronesi U: Axillary dissection can be avoided in selected breast cancer patients: analysis of 401 cases. Anticancer Res 1996: 16: 3913

(14) Greenatl MJ: How should the axilla be treated in breast cancer? Why I favour axillary node sampling in the management of breast cancer. Eur J Surg Oncol 1995:21:2.

(15) Goldhirsch A, Glick JH, Gelber RD, Senn HJ: Meeting highlights: International consensus panel on the treatment of primary breast cancer. J Natl Cancer Inst 1998: 90: 1601 .

(16) Haffty BG, Ward B. Pathare P. Salem R, McKhann C, Beinfield M. Discher D. Reiss M: Reappraisal of the role of axillary lymph node dissection in the conservative treatment of breast cancer. J Clin Oncol 1997: 15:691.

(17) Halverson KJ. Taylor ME, Perez CA, Garcia DM. Myerson R. Philpott G. Levy J, Simpson JR. Tucker G, Rush C: Regional nodal management and patterns of failure following conservative surgery and radiation therapy for stage I and II breast cancer. Int J Radiat Oncol Biol Phys 1993: 26: 593.

(18) Harder F, Zuber M, Kocher T, Torhorst J: Endoscopic surgery to the axilla-a substitute for conventional axillary clearance? Rec Results Cancer Res 1998; 152: 180

(19) Harris JR. Lippman ME, Veronesi U, Willett W: Breast cancer (3). N Engl J Med 1992; 327:473.

(20) Kjaergaard J, Blichert-Toft M, Andersen JA. Rank F, Pedersen BV, Danish Breast Cancer Cooperative Group: Probability of false negative nodal staging in conjunction with partial axillary dissection in breast cancer. Br J Surg 1985:72: 365 . (21) Kocher T. Zuber M. Harder F: Die minimal-invasive Chirurgie beim Mammakarzinom. Ther Umsch 1997: 54: 540.

(22) Miller BA. Feuer EJ, Hankey BF: Recent incidence trends for breast cancer in women and the relevance of early detection: an update. CA Cancer J Clin 1993; 43: 27.

(23) Norrow $M$ : Role of axillary dissection in breast cancer management. Ann Surg Oncol 1996; 3: 233

(24) Morrow M: A Survival benefit from axillary dissection: Was Halsted correct? Ann Surg Oncol 1999; 6: 17

(25) Morton DL. Wen DR, Wong JH, Economou JS. Cagle LA. Storm FK. Foshag LJ, Cochran AJ: Technical details of intraoperative lymphatic mapping for early stage melanoma. Arch Surg 1992; 127: 392.

(26) Noguchi S, Aihara T, Motomura K, Inaji H. Imaoka S, Koyama H: Detection of breast cancer micrometastases in axillary lymph nodes by means of reverse transcriptase-polymerase chain reaction. Comparison between MUCI mRNA and keratin 19 mRNA amplitication. Am J Pathol 1996: 148:649.
(27) Ravdin PM. Delaurentiis M. Vendely T. Clark GM: Prediction of axillary lymph node status in breast cancar patients by use of prounostic indicators. I Nat Cancer inst 1994: 86: 1771.

(28) Recht A, Pierce SM. Abner A. Vicini F. Osteen RT, Love SM, Silver B. Harris JR: Regional nodal failure after conservative surgery and radiotherapy for earlystage breast carcinoma. J Clin Oncol 1991:9:988.

(29) Recht A. Houlihan MJ: Axillary lymph nodes and breast cancer: A review. Cancer 1995; 76: 1491

(30) Ridings P. Bucknall TE: Modern trends in breast cancer therapy: towards less ymphoedema. Eur J Surg Oncol 1998: 24: 21

(31) Salvat J, Knopf JF Ayoubi JM. Slamani L, Vincent-Genod, Guilbert M. Walker D: Endoscopic exploration and lymph node sampling of the axilla. Preliminary findings of a randomized pilot study comparing clinical and anatomo-pathological results of endoscopic axillary lymph node sampling with traditional surgical triatment. Eur J Obstet Gynecol Reprod Biol 1996; 70: 165.

(32) Silverstein MJ, Gierson ED, Waisman JR. Senofsky GM. Colburn WJ Gamagami P: Axillary lymph node dissection for Tla breast carcinoma. Is it indicated? Cancer 1994; 73: 664

(33) Smith IC, Ogston KN, Whtiford P, Smith FW. Sharp P. Norton M, Miller ID, Ah-See AK, Heys SD, Jibril JA, Eremin O: Staging of the axilla in breast cancer. Ann Surg 1998: 228: 220.

(34) Suzanne F, Anton MC. Ducroz F, Wattiez A. Fatton B, Jacquetin B: Le curage axillaire dans le cancer du sein par aspiration graisseuse et ganglionnaire: à propos de 57 cas. Ref Gynecol Obstet 1994; 3: 255.

(35) Suzanne F. Wattiez A. Bournazeau JA: Le curage conservateur endoscopique après liposuccion axillaire. Ref Gynecol Obstet 1996: 4: 25.

(36) Tsangaris TN. Trad K. Brody FJ, Jacobs LK. Tsangaris NT, Sackier JM: Endoscopic exploration and sentinel lymphadenectomy. Surg Endosc 1999; 13: 43.

(37) Warmuth MA. Bowen G, Prosnitz LR. Chu L, Broadwater G, Peterson B, Leight G, Winer EP: Complications of axillary lymph node dissection for carcinoma of the breast. Am Cancer Soc 1998: 83:1362.

(38) White RE, Vezeridis MP, Konstadoulakis M. Cole BF, Wanebo HJ, Bland KI Therapeutic options and results for the management of minimally invasive carcinoma of the breast: influence of axillary dissection for treatment of $\mathrm{T} / \mathrm{a}$ and $\mathrm{T} \mathrm{lb}$ lesions. J Am Coll Surg 1996; 183: 575.

(39) Wilmot C, Watemberg S. Landau O. Litwin D: Of balloon axilloscopy and avoidance of iatrogen injury of the long thoracic nerve. Arch Surg 1997; 132: 1 121 . (40) Zuber M, Kocher T, Oertli D, Gambazzi F. Müller D, Köchli O, Torhorst J, Harder F: Das ,sentinel lymph node"-Verfahren als Selektionskriterium für die axilläre Lymphknotendissektion beim Mammakarzinom. In Laffer U. Oertli D (eds): Ethik. Technik und Konzepte: 10 Jahre später. in Laffer U. Oertil D, Dürig M, Harder F (eds): Basler Beiträge zur Chirurgie 1998; 10: 209.

\section{Kongreßankündigung / Congress Announcement}

\section{ACO-Schwerpunktsymposium „Ösophagus und Hypopharynx“}

Ort:

Termin:

Organisation und Information:

\author{
St. Veit an der Glan/Kärnten
}

7. bis 9 . September 2000

Prof. Dr. E. Wenzl, Klinische Abteilung für Allgemeinchirurgie, Universitätsklinik für Chirurgie, Währinger Gürtel 18-20, A-1090 Wien

Tel: $++43 / 1 / 40400-5621$, Fax $++43 / 1 / 40400-6927$

E-mail: ACO_2000@akh-wien.ac.at 